\title{
Earth's Stratospheric Aerosol Parameters Reconstruction from Polarimetric Measurements of the Sky
}

\author{
PETRO NEVODOVSKYI', OLEKSANDR OVSAK' ${ }^{1}$, ANATOLIY VIDMACHENKO', \\ OREST IVAKHIV ${ }^{2}$, OLEKSANDR ZBRUTSKYI ${ }^{3}$, MYKHAYLO GERAIMCHUK ${ }^{3}$ \\ ${ }^{1}$ Main Astronomy Observatory of NAS of Ukraine, Kyiv, Ukraine, (e-mail: nevod@ mao.kiev.ua) \\ ${ }^{2}$ Lviv Polytechnic National University, Lviv, Ukraine, (e-mail: orest.v.ivakhiv@lpnu.ua) \\ ${ }^{3}$ National Technical University of Ukraine "Igor Sikorsky Kyiv Polytechnic Institute", Kyiv, Ukraine, \\ (e-mail: zbrutsky@cisavd.kpi.ua, geraimchuk@kpi.ua) \\ Corresponding author: Orest Ivakhiv (e-mail: orest.v.ivakhiv@1pnu.ua).
}

\begin{abstract}
: ABSTRACT Earth's climate changes are the result of natural changes in the energy balance of Sun irradiation and influence of anthropogenic factors on the variations of ozone layer thickness and stratospheric aerosol abundance. It is developed a miniature polarimeter for satellite polarimetric experiments in the ultraviolet region of the sunlight spectrum. The main task of this device is to the obtain an information on the stratospheric aerosol physical properties. We tested this polarimeter on a bench specially designed and manufactured as well. It is possible to measure by it the phase dependences of the degree of linear polarization (DLP) of solar radiation scattered by the Earth's atmosphere. A set of special computer programs was developed for comparing the spectral polarimetric measurements DLP data of cloudless sky with model calculations of DLP for the artificial gas-aerosol medium. Thus, the prototype of satellite polarimeter as well as special computer programs make it possible to study the Earth's atmosphere aerosol physical characteristics.
\end{abstract}

KEYWORDS remote observation; aerosol; polarimeter; satellite.

\section{INTRODUCTION}

$\mathrm{W}$ eather and climate change on Earth is the result of natural changes in the energy balance of solar radiation. Changes in the power of the stratospheric aerosol affect changes in the Earth's temperature balance both directly and through participation in the formation and destruction of ozone molecules, which protect our Earth from harsh ultraviolet radiation.

A lot of polarimetric experimental projects in the ultraviolet (UV) sunlight spectral region were repeatedly proposed for stratospheric aerosol investigation by the Main Astronomy Observatory of the NAS of Ukraine (see, for example, $[1,2])$.

There is another method $[3,4]$ for obtaining information on the physical properties of stratospheric aerosols of the planets of the solar system using observations in the visible and near infrared spectral wavelengths.
We plan to obtain them using polarimetric measurements of the twilight sky by the technology proposed in [5-7].

To determine the albedo of single scattering and refine the size of aerosol particles, it is necessary to carry out photometric measurements of the corresponding objects [8].

The information obtained in the past half of the 20-th century was given in [9]. Our measurements on different days without filters showed that the degree of linear polarization (DLP) at the zenith of the sky is $30-70 \%$ [10]. This is much less than the DLP value for pure gas atmospheres, which often goes up to $100 \%$. There is practically no dependence of DLP on the height of the scattering layer. There is also a slight decrease in DLP, because of possible sky background depolarizing effect. With an increase in the solar zenith distance of more than 
$90^{\circ}$, the sky DLP value decreases. It also increases with increasing of wavelength. It was noted in $[1,11]$ that the influence of a large aerosol amount in the troposphere increases strongly at wavelengths exceeding $760 \mathrm{~nm}$. The aerosol DLP at the $90^{\circ}$ phase angle was estimated near the $20 \%$. Since the end of 2006, a slight increase in the amount of stratospheric aerosol has been detected. This is evidenced by a decrease in DLP at a wavelength of $525 \mathrm{~nm}$ at solar zenith angles $91-94^{\circ}$.

Such a decrease in polarization is possible to explain as an influence of Rubal volcano eruption in New Guinea (October 2006). Due to it, a significant amount of dust was released into the atmosphere.

Aerosol can stay quite long in the stratosphere at altitudes of more than $30 \mathrm{~km}$. It significantly affects the Earth thermal regime as well as the ozone layer thickness. The data of aerosol optical thickness at such height was obtained by spectrophotometric studies [3, 12-14]. But the real part of the refractive index, the particles size distribution function type and parameters, the horizontal structure, the ozone layer altitude position, etc. are not completely clear, yet. At the same time, polarization measurements made it possible to determine such characteristics most correctly among remote methods.

The proper measurements can be performed using spacecraft observations, the ground-based stations and / or both of them. The papers $[15,16]$ describe the polarimetric experiments of studying stratospheric aerosol in the ultraviolet region of the spectrum for the atmospheres of the planets Venus and Mars, where these problems were successfully solved. We proposed to conduct a similar space experiment to study the stratospheric aerosol of the Earth, for which the operating model of the UVP was made. However, preparation for the implementation of our experiment is proceeding slowly.

Therefore, a test ground experiment was carried out to assess the operation of the measuring system and the possibility of testing the method for studying the polarization component of a cloudless twilight sky described in [4]. The conducted work showed that we can carry out studies of a particular altitude level of the atmosphere [16] planning observations either in winter (with declination $\sigma=-23^{\circ}$ ) or in summer (with declination $\sigma=+23^{\circ}$ ).

\section{THE DESCRIPTION OF OUR DEVICE AND THE METHODOLOGY OF OBSERVATIONS}

We prepared an experiment to study the physical characteristics of stratospheric aerosol from a spacecraft. As a result, a mock-up of an ultraviolet polarimeter (UVP) has been [2] was created, which makes it possible to remotely measure the polarization components of changes in the Earth's stratosphere from a spacecraft.

The photomultiplier R1893 operates at the photoncounting mode. Temperature sensors check a temperature of both the medium and the receiver. A special designed hollow-rotor piezoelectric motor rotates the polarization element. Necessary data from temperature sensors (on the receiver, on the engine, etc.) are transferred to computer interface for the purpose of processing and further analysis. The special software was developed for controlling the plane of polarization of the corresponding elements position control, respectively to the put target.

A special bench to set up and study the current prototype of the UVP, its individual blocks and their combination, was developed in [2] (see Fig. 1). It can be divided into the separate interchangeable parts, units and blocks.

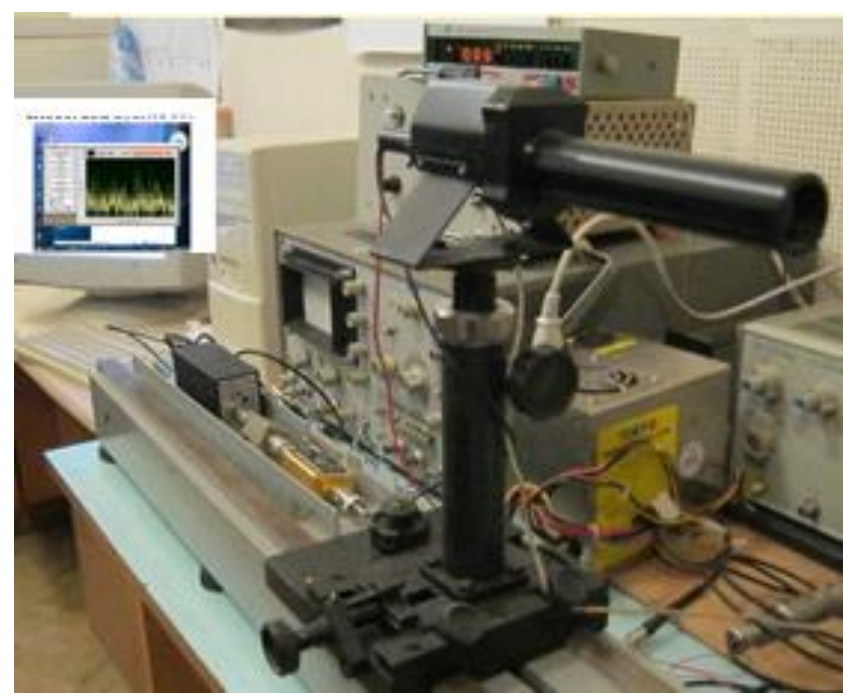

Figure 1. Mockup of on-board ultraviolet polarimeter at the stand

Such a design makes it possible to change research tasks easily and quickly as well as constantly improve the bench itself. The UVP studies performed at the test bench made it possible to determine its technical parameters and performance characteristics. With this equipment, the operation of the light emission receiver (R1893 photomultiplier) was thoroughly investigated to determine its noise threshold at supply voltages in the $1050-1500 \mathrm{~V}$ range (dark pulses are 2-4 pulses/sec) and the device operating voltage was selected. Reading stability of device output signals was investigated extensively. It was determined during long hours of dark and useful signals measurements. To verify the methodology of carrying out polarization observations [10], 17-19] of the cloudless sky, we used a modification of the current prototype of the onboard ultraviolet polarimeter UVP $[2,10]$. We mounted it on the AZT-2 telescope ( $70 \mathrm{~cm}$ mirror and $15 \mathrm{~m}$ focal length) (see Fig. 2). We did the polarimetric observations with improved prototype of ultraviolet polarimeter on 26-th and 27-th of September 2017. We oriented the telescope at a part of the sky with the Sun declination equal to $\delta \approx 0^{\circ}$ for an angle equal to $1 \mathrm{~h}$ from the central meridian. Operation began by turning off the clockwork mechanism of the telescope at 14:00 UTC+2 and observations were completed at 20:00. For the observations we used the $\lambda=362 \mathrm{~nm}$ filter located between the visible and ultraviolet light spectral regions and 
selected the sky region using a diaphragm with a diameter of $0.5 \mathrm{~mm}$.

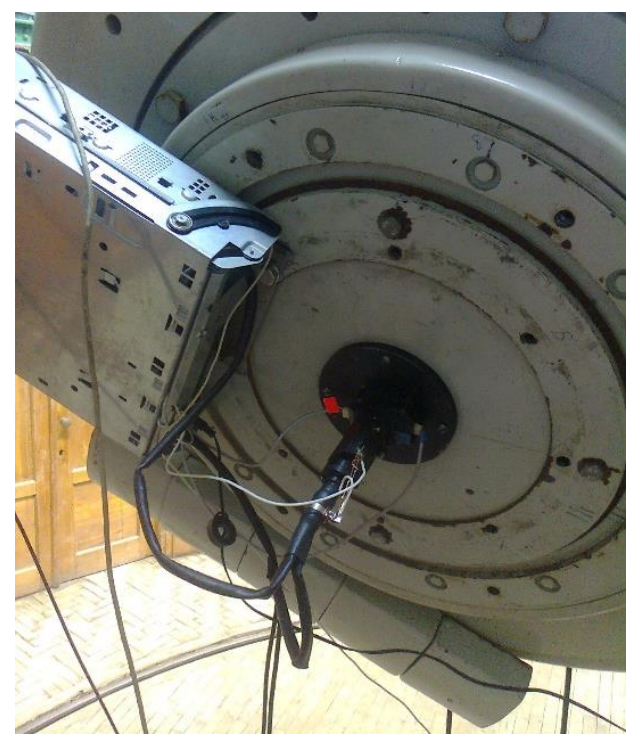

Figure 2. Ultraviolet polarimeter at the AZT-2 telescope.

The piezoelectric motor rotates the modulator unit with the polarization element (Glan prism) at a $45^{\circ}$ the same angle. Thus, one rotation through $360^{\circ}$ the modulator carried out in 8 steps. Exposure was selected in 2 seconds. At the beginning of operation, the useful signal stream was about $300 \mathrm{k}$ pulses per second with a dark stream of no more than 20 pulses per second. The results of obtained observations [10], as well as the additional results [20] can be used for analysis and subsequent processing.

\section{COMPUTATIONAL ALGORITHM FOR SPECTRAL POLARIMETRY MEASUREMENTS DATA OF CLOUDLESS SKY}

The linear polarization degree of solar radiation scattered by Earth's atmosphere is determined by the combined effect of light scattering on gas molecules and aerosol particles [21]. A phase dependence of the molecular (Rayleigh) part of sky DLP $P_{R}(\alpha)$ is represented as follows:

$$
P_{R}(\alpha)=\sin ^{2}(\alpha) /\left(1+\cos ^{2}(\alpha)\right)
$$

where $\alpha$ is the phase angle; $P_{R}^{\prime}(\alpha)$ is the Rayleigh component of the DLP, calculated taking into account the influence of the depolarization factor and multiple scattering.

With a small atmospheric spectral optical thickness $\tau(\lambda)<<1$, the DLP of light scattered by gases once has a maxima at both phase angles equal to $90^{\circ}$ and $270^{\circ}$. The DLP of light scattered by atmosphere gas component decreases due to the depolarizing effect of multiple scattering in case of significant increase in the value of $\tau(\lambda)$. When sunlight is scattered by aerosols, the DLP has complex phase dependence and it takes both a positive and negative values. Namely, the magnitude of the DLP of light depends on wavelength $\lambda$, the nature of the particles, their shapes, the type and parameters of size distribution function (average $r_{0}$ or effective radius $r_{e f}$ and their dispersion), as well as on the phase angle [22-25].

The DLP phase dependence of light scattered by small isotropic particles with low true absorption is close to Rayleigh scattering. However, if the imaginary part of complex refractive index of those particles increases significantly, the maxima of the DLP from the phase angles of $90^{\circ} / 270^{\circ}$ are shifted toward values equal to $60^{\circ} / 300^{\circ}$, respectively. In case the aerosol physical parameters are unchanging, the DLP of the light scattered by particles decreases with wavelength decreasing. There were modeled the characteristics of light scattering by a static ensemble of homogeneous randomly oriented particles, having the simplest non-spherical shape (for example, elongated and oblate spheroids, cylinders). Results of such works showed a significant effect of particle shapes on the radiation characteristics scattered by them.

However, attempts to simulate the scattering characteristics of aerosol particles with complex shapes that exist in nature (crystals, snowflakes, soot, dust, etc.) encounter the impossibility of an analytical description and the complexity of the algorithmizing of such a problem. Researchers must use computer clusters with huge RAM resources and extremely high speed in addition.

The spectral atmospheric DLP value $P(\alpha, \lambda)$ can be represented as follows:

$$
P(\alpha, \lambda)=\beta(\lambda) \cdot P_{R}^{\prime}(\alpha)+(1-\beta(\lambda)) \cdot P_{a}(\alpha, \lambda, \rho, m),
$$

where $P_{a}(\alpha, \lambda, \rho, m)$ is an aerosol component of the DLP; $\rho=2 \pi a / \lambda$ is the Mie parameter, $a$ is the particle radius, $\beta(\lambda)$ is the spectral value of molecular scattering relative contribution:

$$
\beta(\lambda)=\sigma_{R}(\lambda) /\left[\sigma_{R}(\lambda)+\sigma_{a}(\lambda)\right]
$$

where $\sigma_{\mathrm{R}}(\lambda)$ is the gas and $\sigma_{\mathrm{a}}(\lambda)$ is the aerosol volumetric spectral scattering coefficient, respectively.

We have determined the spectral values quantity $\beta(\lambda)$ using the expression (3), modified to take into account the possible multimode composition of the investigated atmosphere aerosol component:

$$
\beta(\lambda)=\left[\frac{\left(k_{1} \sigma_{a 1}(\lambda)+k_{2} \sigma_{a 2}(\lambda)+\ldots+k_{n} \sigma_{a n}(\lambda)\right)}{\left(k_{1} \sigma_{a 1}\left(\lambda_{0}\right)+k_{2} \sigma_{a 2}\left(\lambda_{0}\right)+\ldots+k_{n} \sigma_{a n}\left(\lambda_{0}\right)\right)} \times\left(\frac{\lambda}{\lambda_{0}}\right)^{4} \cdot\left(\frac{1-\beta\left(\lambda_{0}\right)}{\beta\left(\lambda_{0}\right)}\right)+1\right]^{-1},
$$

where $\sigma_{a i}(\lambda), k_{i}$ are the volume scattering coefficient and its weight coefficient in the total aerosol volume for the $i$-th 
aerosol mode, respectively; $\beta\left(\lambda_{0}\right), \sigma_{a i}\left(\lambda_{0}\right)$ are the values of corresponding quantities at the wavelength $\lambda_{0}$.

DLP aerosol component $P_{a}(\alpha, \lambda, \rho, m)$ is formed by both single and multiple scattering processes. The multiple light scattering can be neglected in the long wavelength part of the sunlight wavelength range. However, this must be on consideration on short wavelengths, where its influents value increases significantly.

A set of special computer program codes were developed by us to simulate the spectral phase atmospheric DLP dependencies. There was used a model of polydisperse homogeneous spherical aerosol particles ensemble with a normal-logarithmic size distribution function.

The degree of linear polarization, scattering coefficient, and phase function of model aerosol particles are calculated according to a technique developed using the results of $[8$, $17,26-45]$.

We calculate spectral phase dependences of DLP on the light scattered by a model homogeneous gas-aerosol medium in a physically acceptable range of aerosol parameters using our software package. The specified accuracy of the calculations is equal to $10^{-8}$.

\section{A SAMPLE RESULTS OF OBSERVATIONAL DATA} ANALYSIS

There were analyzed spectral phase angle dependencies of the sky linear polarization degree. Figures $3 \mathrm{a}, \mathrm{b}, \mathrm{c}$ are plotted from measurements data at the zenith of the sky above Kyiv [20].

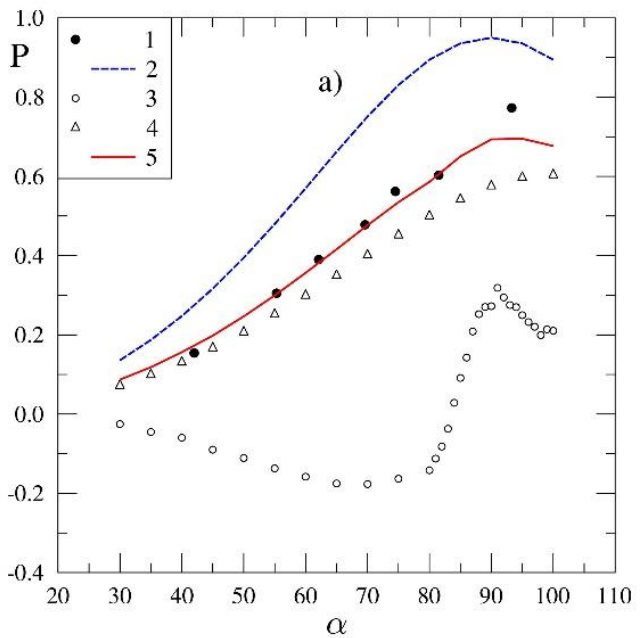

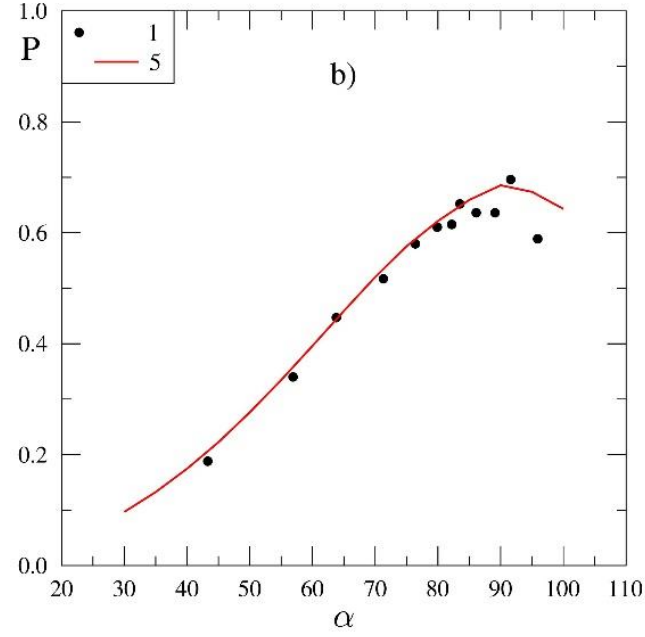

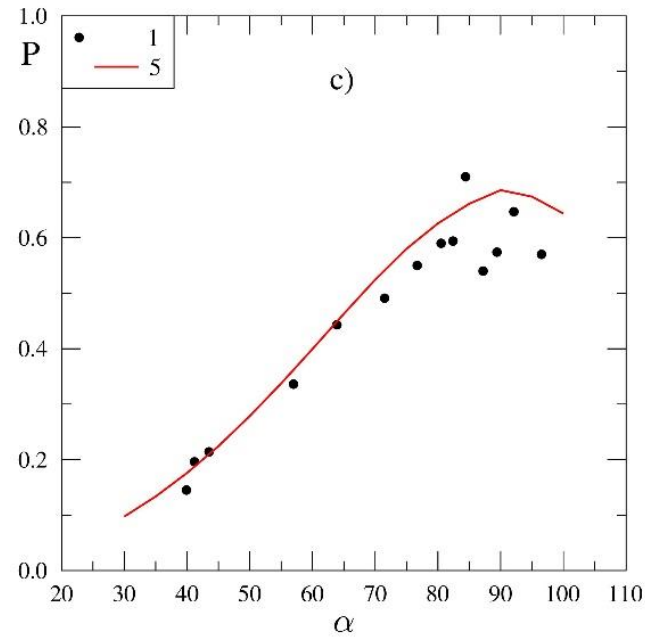

Figure 3. Phase angle dependences of the sky zenith DLP (Kyiv, Ukraine), namely: 1 is the sky DLP measurements data from [20]; 2 is the DLP of simulated Rayleigh scattering; 3 is the DLP of simulated aerosol mode $1 ; 4$ is the DLP of aerosol mode 2; 5 is the result of adding the calculated components 2,3 , and 4 .

Here were used theoretical characteristics calculated separately for the gas and for the two recovered aerosol component modes, as well as the result of their summation at wavelengths of $\lambda=578 \mathrm{~nm}, \lambda=420 \mathrm{~nm}$ and $\lambda=390 \mathrm{~nm}$, respectively.

These results allowed us to conclude that polydisperse ensemble of aerosol particles with at least two different aerosol fractions (modes) was probably presented on the date of measurements in the sky above Kyiv [20]. Certain aerosol parameters for the normal-logarithmic particle sizes distribution function are shown in Table 1. 
Table 1. Certain aerosol parameters for the normal-logarithmic particle sizes distribution function

\begin{tabular}{|l|l|l|c|}
\hline \multicolumn{1}{|c|}{ The mode type } & $\begin{array}{c}\text { The real part of particles refractive } \\
\text { index }\end{array}$ & $\begin{array}{c}\text { The geometric average particles } \\
\text { radius }\end{array}$ & The radius dispersion value \\
\hline $\begin{array}{l}\text { The first mode 1 has a weight } \\
\text { coefficient equal to } \\
k_{1}=0.22\end{array}$ & $n_{r 1}=1.45+0.02 /-0.01$ & $r_{01}=6.7+2.4 /-1.4 \mu m$ & $\sigma^{2}=0.1 \pm 0.02$ \\
\hline $\begin{array}{l}\text { The second mode 2 of finely } \\
\text { dispersed particles }\end{array}$ & $n_{r 2}=1.45 \pm 0.01$ & $r_{02}=0.11 \pm 0.005 \mu m$ & $\sigma^{2}=0.1 \pm 0.005$ \\
\hline
\end{tabular}

Spectral values of gas scattering relative contribution parameter are as follows:

$$
\begin{gathered}
\beta(578 \mathrm{~nm})=0.45, \beta(420 \mathrm{~nm})=0.61 \text { and } \\
\beta(390 \mathrm{~nm})=0.64 .
\end{gathered}
$$

These results are in good matching with results of the published measurements in [46].

\section{CONCLUSION}

A miniature satellite ultraviolet polarimeter is developed for the stratospheric aerosol characteristics studies. We have determined its technical parameters and performance characteristics on the special bench. The polarimeter prototype modification is tested on the AZT telescope. An aerosol influence on spectral and phase dependencies of sky polarization properties is shown. The special computer programs are developed for cloudless sky polarization characteristics analysis. A sample analysis of observational data is demonstrated.

\section{References}

[1] O. S. Ugolnikov, I. A. Maslov, "Studies of the stratosphere aerosol layer based on polarization measurements of the twilight sky," Cosmic Research, vol. 47, issue 3, pp. 198-207, 2009. https://doi.org/10.1134/S0010952509030022.

[2] P. V. Nevodovskyi, O. V. Morozhenko, A. P. Vidmachenko, O. Ivakhiv, M. Geraimchuk, O. Zbrutskyi, "Tiny ultraviolet polarimeter for earth stratosphere from space investigation," Proceedings of the IEEE 8th International Conference on Intelligent Data Acquisition and Advanced Computing Systems: Technology and Applications (IDAACS'2015), 24-26 September 2015, Warsaw, Poland, vol. 1, pp. 28-32. https://doi.org/10.1109/IDAACS.2015.7340695.

[3] O. V. Morozhenko, A. V. Shavrina, O. A. Veles', "Conception of gas and aerosol pollution monitoring of the earth's atmosphere (for altitudes more than $30 \mathrm{~km}$ ) on board the international space station," Cosmic Science and Technology, vol. 6, no. 2/3, pp. 69-76, 2000. https://doi.org/10.15407/knit2000.02.069.

[4] A. V. Morozhenko, A. P. Vidmachenko, P. V. Nevodovskii, "Aerosol in the upper layer of earth's atmosphere," Kinematics and Physics of Celestial Bodies, vol. 29, no. 5, pp. 243-246, 2013. https://doi.org/10.3103/S0884591313050073.

[5] O. S. Ugolnikov, I. A. Maslov, "Stratospheric aerosol particles size distribution based on multi-color polarization measurements of the twilight sky," Journal of Aerosol Science, vol. 117, pp. 139-148, 2018. https://doi.org/10.1016/j.jaerosci.2018.01.002.

[6] O. S. Ugolnikov, I. A. Maslov, "Optical depths and vertical profiles of stratospheric aerosol based on multi-year polarization measurements of the twilight sky," Journal of Aerosol Science, vol. 127, pp. 93-101, 2019. https://doi.org/10.1016/j.jaerosci.2018.10.006.

[7] O. S. Ugolnikov, "Twilight sky photometry and polarimetry: the problem of multiple scattering at the twilight time," Cosmic Investigations, vol. 37, no. 2, pp. 168-175, 1999.
[8] M. F. Sterzik, S. Bagnulo, D. M. Stam, et al., "Spectral and temporal variability of Earth observed in polarization," Astronomy \& Astrophysics, vol. 622, id. A41, 19 p., 2019. https://doi.org/10.1051/0004-6361/201834213.

[9] G. V. Rozenberg, Twilight, Moscow: Fizmatgis, 1963, 380 p. (in Russian).

[10] P. V. Nevodovskyi, O. V. Morozhenko, A. P. Vidmachenko, O. Ivakhiv, O. Zbrutskyi, M. Geraimchuk, "Reliable stratospheric aerosol of the Earth's atmosphere observations," Proceedings of the IEEE 9th International Conference on Dependable Systems, Services and Technologies (DESSERT'2018), 24-27 May 2018, Kyiv, Ukraine, pp. 667-672. https://doi.org/10.1109/DESSERT.2018.8409208.

[11] O. Dubovik, Zh. Li, M. I. Mishchenko, et al. "Polarimetric remote sensing of atmospheric aerosols: Instruments, methodologies, results, and perspectives," Journal of Quantitative Spectroscopy and Radiative Transfer, vol. 224, pp. 474-511, 2019. ttps://doi.org/10.1016/j.jqsrt.2018.11.024.

[12] O. Bazhenov, V. Burlakov, S. Dolgii, A. Nevzorov, N. Salnikova, "Optical monitoring of characteristics of the stratospheric aerosol layer and total ozone content at the Siberian Lidar Station (Tomsk: $56^{\circ}$ $30^{\prime} \mathrm{N} ; 85^{\circ} \mathrm{E}$ )," International Journal of Remote Sensing, vol. 36, issue $11, \quad$ pp. 3034-3042, 2015. https://doi.org/10.1080/01431161.2015.1054964.

[13] G. Taha, P.K. Bhartia, Z. Chen, P. Xu, R.P. Loughman, G. Jaross, "OMPS limb profiler: Extending SAGE and CALIPSO stratospheric aerosol records," Proceedings of the American Geophysical Union, Fall Meeting 2017, 2017, abstract \#A41O-07.

[14] J. Hansen, "Long-term monitoring of global climate forcing and feedbacks," Proceedings of the Conference on Climate Forsings and Feedbacks, Ed. by J. Hansen, W. Rossow, I. Fung, New York, 1992, pp. 6-12.

[15] J. E. Hansen, A. Arking, "Clouds of Venus: Evidense for their nature," Science, vol. 171, no. 3972, pp. 669-672, 1971 https://doi.org/10.1126/science.171.3972.669.

[16] C. Gebhardt, A. Abuelgasim, "Scientific innovations from the Mars aerosol optical depth based on satellite data with a temporal resolution of hours," Progress in Earth and Planetary Science, vol. 6, Article number: 31, 2019.

[17] E. G. Yanovitskij, Z. O. Dumanskij, Tables on the Scattering of Light of a Polydisperse System of Spherical Particles, Kiev: Naukova dumka, 1972, 124 p. https://doi.org/10.1186/s40645-019-0276-Z.

[18] M. I. Mishchenko, B. Cairns, J. E. Hansen, L. D. Travis, R. Burg, J. Kaufman, J. Vanderlei Martins, E. P. Shettle, "Monitoring of aerosol forcing of climate from space: analysis of measurement requirements," Journal of Quantitative Spectroscopy \& Radiative Transfer, vol. 88, pp. 149-161, 2004. https://doi.org/10.1016/j.jqsrt.2004.03.030.

[19] "Errors in UV reflectivity and albedo calculations due to neglecting polarization", Piet Stammes Royal Netherlands Meteorological Institute (KNMI), P.O. Box 201, 3730 AE De Bilt, The Netherlands.

[20] V. V. Avramchuk, Multicolor Polyrimetry of Light of Twilight and Daytime Sky at Zenith, Voprosy astrofiziki, Kiev: Naukova dumka, 1965, pp. 112-120. (in Russian).

[21] D. L. Coffeen, "Polarization and scattering characteristics in the atmospheres of Earth, Venus, and Jupiter," Journal of the Optical Society of America, vol. 69, issue 8, pp. 1051-1064, 1979. https://doi.org/10.1364/JOSA.69.001051.

[22] A. Mugnai, W.J. Wiscombe, "Scattering at radiation by moderately nonspherical particles," J. Atmos. Sci., vol. 37, no. 6, pp. 1291-1307, 1980. 0469(1980)037<1291:SORBMN>2.0.CO;2. https://doi.org/10.1175/1520- 
[23] M. I. Mishchenko, "Light scattering by randomly oriented axially symmetric particles," J. Opt. Soc. Amer., vol. 8, no. 5, pp. 871-882, 1991. https://doi.org/10.1364/JOSAA.8.000871.

[24] M. I. Mishchenko, L. D. Travis, D. W. Mackowski, "T-matrix computations of light scattering by nonspherical particles: a review," Ibid, vol. 55, no. 5, pp. 535-575, 1996. https://doi.org/10.1016/00224073(96)00002-7.

[25] J. Takahashi, Y. Itoh, H. Akitaya, et al., "Phase variation of earthshine polarization spectra," Publications of the Astronomical Society of Japan, vol. 65, no. 2, id. 38, pp. 1-9, 2013. https://doi.org/10.1093/pasj/65.2.38.

[26] D. Deirmendjian, Electromagnetic scattering on spherical polydispersions, New York: Elsevier Scientific Publishing, 1969, 290 p.

[27] C. Emde, R. Buras-Schnell, M. Sterzik, S. Bagnulo, "Influence of aerosols, clouds, and sunglint on polarization spectra of Earthshine," Astronomy \& Astrophysics, vol. 605, id. A2, 15 p., 2017. https://doi.org/10.1051/0004-6361/201629948.

[28] G. van de Hulst, Light Scattering by Small Particles, New York: John Wiley and Sons, London: Chapman and Hall, 1957.

[29] P.A. Miles-Páez, E. Pallé, M.R. Zapatero Osorio, "Simultaneous optical and near-infrared linear spectropolarimetry of the earthshine," Astronomy \& Astrophysics, vol. 562, id. L5, 4 p., 2014. https://doi.org/10.1051/0004-6361/201323009.

[30] M. Yaknik, W. Heller, J. Witeczek, Tables of Angular Scattering Functions for Heterodisperse Systems of Spheres, Wayne State University Press (May 22, 1969), 1308 p.

[31] O. S. Ovsak, V. M. Vashenko, A. P. Vid'machenko, Ye. A. Loza, Zh. I. Patlashenko, B. O. Ovsak, "Recovery of parameters for the multimodal aerosol component in the atmosphere from spectral polarimetric measurements," Ukr. J. Phys., vol. 66, no. 6, pp. 466-477, 2021. https://doi.org/10.15407/ujpe66.6.466.

[32] D. Huige, W. Qiyu, H.Hangbo, L. Siwen, Y. Qing, L. Jingjing, S Yuchui, H. Dengxin. Aerosolmicrophisical particle parameter inversion and error analysis based on remote sencing data," Comput. Sci. Geol.-Remote Sens., 10, 1753, 2018. https://doi.org/10.3390/rs10111753.

[33] D. Huige, H. Hua, Y. Cui, D. Hua, T. He, Y. Wang, Q. Yan. "Vertical distribution of optical and microphysical properties of smog aerosols measureds by multi-wavelength polarization lidar in Xi'an, China," $J$. Quant. $\quad$ Spectrosc. Radiat. Transf., 188,28, 2017. https://doi.org/10.1016/j.jqsrt.2016.05.027.

[34] "Air quality in Europe - 2019 report”, EEA Report No.10/2019, 2019.

[35] O. J. Mousis, D. H. Atkinson, and the Hera Team.”The Hera Saturn entry probe mission. A proposal in response to ESA call for a medium size mission oppor tunity in ESA's science programme for launch in 2019-2030”, 2016, https://arxiv.org/abs/ 1510.07685.

[36] "Earth science and application from space. National imperatives for the next decade and beyond," National Research Council of the National Academies, Washington, DC, 2007.

[37] F. Karagulian, M. Gerbolos, M. Barbiere, A. Kotsev, F. Lagler, A Borowiak, Review of Sensors for Air Quality Monitoring, Publications Office of the European Union, 2019.

[38] https://science-data.larc.nasa.gov/large/

[39] https://science-data.larc.nasa.gov/large/atmosphere.html

[40] https://science-data.larc.nasa.gov/large/instruments.html

[41] https://photojournal.jpl.nasa.gov/gallery/snt

[42] https://www.nasa.gov/subject/3126/air/

[43] http://www.aura.gsfc.nfsa.gov/science

[44] http://www.priroda.su/item

[45] http://www.nasa.gov/topics/earth/features/olympic

[46] V. Bovchalyuk, G. Milinevs'kyi, V. Danylevs'kyi, F. Golub, M. Sosonkin, Yu. Yukhymchuk, T. Podvin, "Properties of an aerosol in the atmosphere over Kyiv according to lidar and photometric observations," Kosm. Nauka Tekhnol., vol. 23, no. 6, 34, 2017. (in Ukrainian). https://doi.org/10.15407/knit2017.06.034.

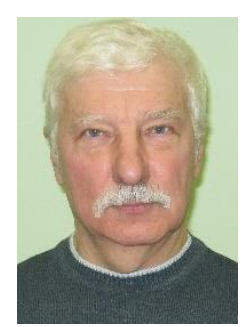

Dr. PETRO NEVODOVSKYI is the Senior researcher of the Main Astronomical Observatory of the National Academy of Sciences of Ukraine. His research interests are corresponded with the investigation of the return atmosphere radiation by polarization methods; development of resources for polarization measuring; elements and systems of polarimeters.

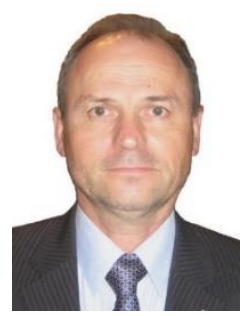

OLEKSANDR OVSAK, PhD in physics and mathematics. Senior Researcher Scientist. Oleksandr Ovsak works currently with the Department for Physics of Sub-stellar and Planetary Systems, Main Astronomical Observatory, National Academy of Sciences of Ukraine. His job title is a Senior Research Officer. Oleksandr does research in aerosols vertical structure of Giant planets atmospheres as well the aerosol modes parameters in the atmosphere of Earth. The main scientific problems that Oleksandr deals with are: Interaction physics of light with a planetary atmosphere; Atmospheric aerosols vertical structure modeling; Radiative transfer theory; Development of the new methods of atmospheric aerosols parameters determination.

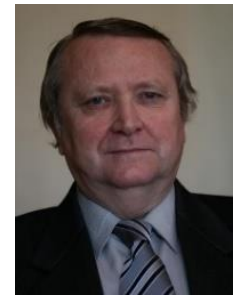

ANATOLIY VIDMACHENKO is the Chief Scientific Researcher of the Main Astronomical Observatory of National Academy of Sciences of Ukraine. Research interests are corresponded with the study of planetary atmospheres and exoplanets characteristics.

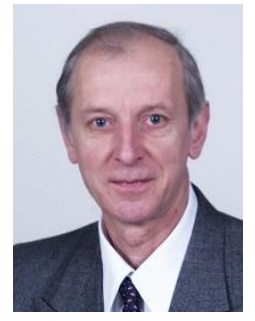

OREST IVAKHIV is from Lviv, Ukraine. Professor O.Ivakhiv received his M.A. Sc. in Electrical Engineering from The Lviv Technical University (Ukraine), the Ph.D. in Communications from The Moscow Aviation Institute (Russia), the Sc. D. in Electrical Instrumentation from Lviv Polytechnic National University (Ukraine). Orest Ivakhiv was a visiting professor at the Department of Electrical and Computer Engineering at The University of Toronto (Ontario, Canada) in 1994. Since 1968 professor O. Ivakhiv is a faculty member at the Computer Technology, Automation and Metrology Institute of Lviv Polytechnic National University. Now Orest Ivakhiv is a Head of the Intelligent Mechatronics and Robotics Department. Orest Ivakhiv has more than 300 publications, 12 patents. His research interests include electrical measurement and instrumentation, informative measurement theory, adaptive data processing, data compression, enumerative coding, communication theory and mechatronics.

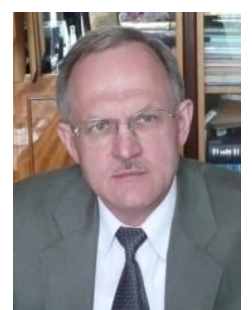

OLEKSANDR ZBRUTSKYI is the Head of Control Systems Department Igor Sikorsky Kyiv Polytechnic Institute, Doctor of Technical Sciences, Professor; Member of Academy of Technology Science, International Academy of Navigation and Movements Control; Honors: State Prize of USSR, State Prize of Ukraine; Honors Professor of North-Western Polytechnic 
University and North China Institute of Aerospace Engineering, China; Coordinator of international Programs TEMPUS, Erasmus; Publication: more than 350, Editor in a chief of scientific journals "Information Systems, Mechanics and Control "(ISSN 2219-3804), "Mechanics of Gyroscopic Systems" (ISSN 0203 - 3771); organizer of the International Scientific and Technical Conference "Gyro-technologies, navigation, motion control and design of aerospace technic". The field of research: integrated navigation systems with technical vision; inertial sensors accuracy increasing, undetermined external disturbances compensation in dynamic control systems; artificial intelligence algorithms for navigation and control systems; dynamic and control of robotics and unman vehicles.

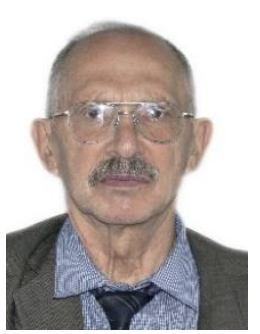

MYKHAYLO GERAIMCHUK, DSc, professor of the Devicemaking Department of the National Technical University of Ukraine "Igor Sikorsky Kyiv Polytechnic Institute", Kyiv, Ukraine. The field of research: sensor networks; inertial sensors, mechatronics and robotics, unmanned vehicles. 